\title{
Synthesis and swelling behavior of poly (acrylic acid-acryl amide- 2-acrylamido-2-methyl-propansulfonic acid) superabsorbent copolymer
}

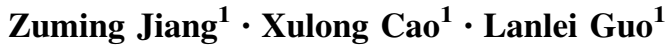

Received: 24 March 2015/Accepted: 13 February 2016/Published online: 27 February 2016

(c) The Author(s) 2016. This article is published with open access at Springerlink.com

\begin{abstract}
Superabsorbent copolymer (SAP) based on acrylic acid, acryl amide and 2-acrylamido-2-methylpropansulfonic acid has been prepared in an aqueous solution using potassium persulfate as initiator and $N, N^{\prime}$ methylenebiacrylamide (NMBA) or triethyleneglycol diacrylate (TEGDA) as crsosslinker. The absorbing properties such as equilibrium water absorbency and swelling properties in different concentration of saline waters were investigated. The relationship between the properties and synthesis conditions such as the concentration and kinds of crosslinking agent, initiators and neutralization degree and monomer ratio were also investigated. The results show that the water absorbency of the SAPs reach $58 \mathrm{~g} / \mathrm{g}$ at the ionic strength of $1000 \mathrm{mmol} / \mathrm{L}$ and the water absorbency decreases with the increase of the concentration of saline water.
\end{abstract}

Keywords Superabsorbent polymer - 2-Acrylamido-2Methyl-Propansulfonic acid · Solution polymerization . Swelling property $\cdot$ Water absorbency

\section{Introduction}

Superabsorbent polymers (SAPs) are one kind of materials which can absorb large amount of water, and they are different from other hydrophilic materials because they can retain water even under pressure. Due to the characteristics, SAPs have been widely used in many fields, such as

Zuming Jiang

zumingjiang@hotmail.com

1 Research Institute of Exploration and Development, SLOF, Dongying 257015, China disposable diapers, women napkins, soil for agriculture and oilfield conformance (Flory 1953; Zou 2002).

The influence of various polymerization parameters on the absorbency or absorption rate has been investigated by many researchers. Chen et al. reported the synthesis of poly (acryl amide-co-acrylic acid) by solution polymerization (Guo and Liu 2005). Y. Murali Mohan reported the synthesis of poly (acryl amide-co-sodium methacrylate) copolymers by free radical polymerization in aqueous solution, and the various reaction parameters had also been investigated (Zhang et al. 2006). Lin et al. reported PAMA prepared in aqueous solution using acryl amide and 2-acryamido-2-methyl-propanesulfane acid as monomers, and the absorbency abilities in different solutions had been studied (Gosavi et al. 1999).

Although many studies have been done, there are still some problems that need to solve. For example, most of SAPs are weakly cross-linked acrylic acid-based polymer and they are often used under salinity conditions, so much more attentions should be focused on the improvement of their salt resistance. Moreover, SAPs are often used under pressure conditions, so the gel strength should also be considered.

As is well known, salt-resistance ability is very important to SAPs and it depends on functional group, crosslinking density and reaction conditions (Chen et al. 2005; Murthy et al. 2006; Mohan et al. 2005; Lee and Huang 2007; Y1lmaz and Kul 2007; Xu et al. 2007; Vandamme and Lenourry 2002; Karadağ and Saraydin 2002; Wu and Lin 2000; Pourjavadi et al. 2007; Li et al. 2007). In this study, with the purpose of improving the salt-resistance ability, a new SAP has been synthesized in aqueous solution using acrylic acid (AA), acryl amide (AM) and 2-acrylmido-2-methylpropanesulfonic acid (AMPS) as monomers. AA and AMPS determine the water absorbency 
Fig. 1 Effect of monomer molar ratio (AA/AM/AMPS) on the water absorbency of SAPS

Fig. 2 Effect of crosslinking agent (NMBA) concentration on the water absorbency of SAPs
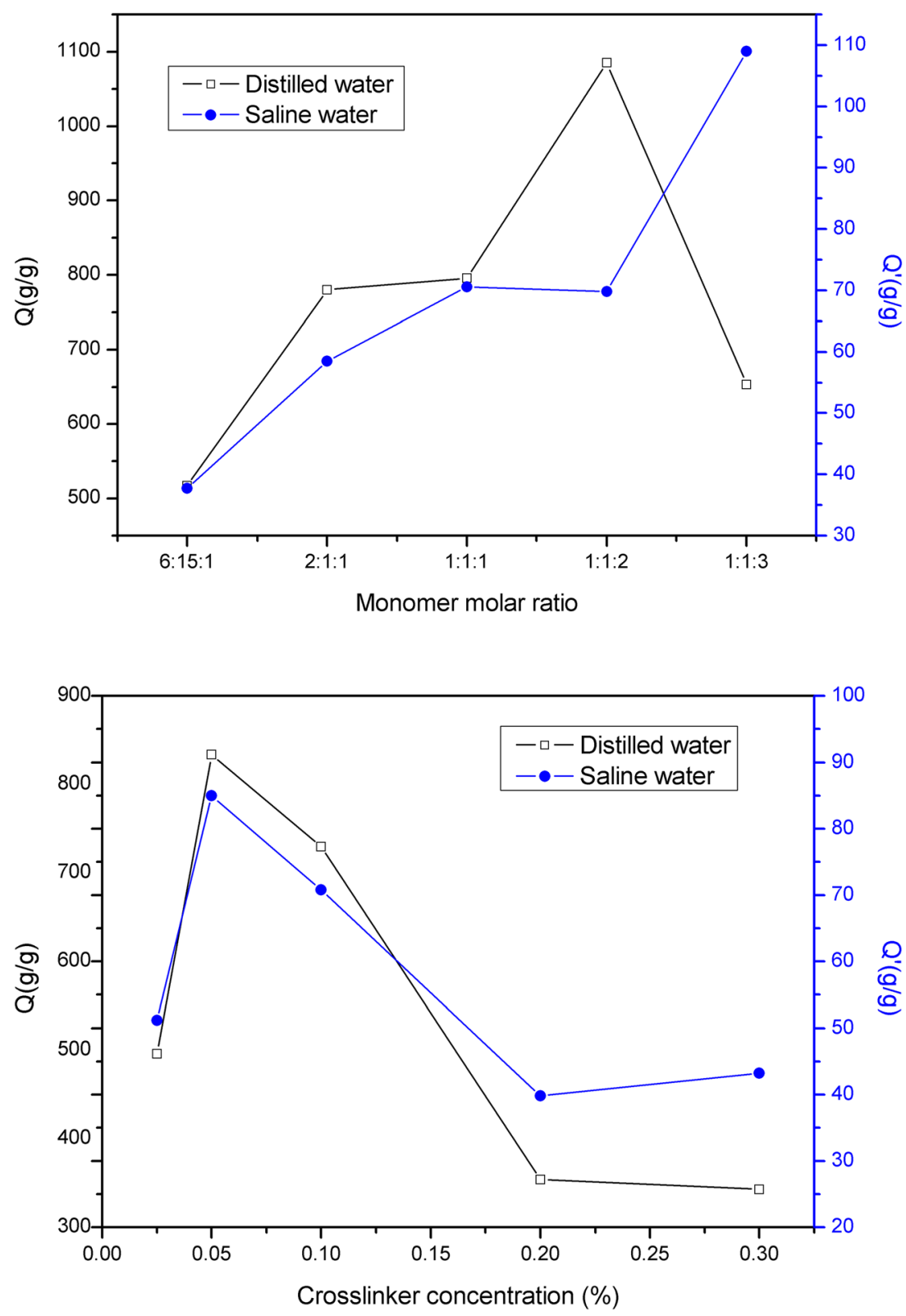

ability, and AM can contribute to the gel strength and reduce the cost. The swelling properties of SAP in distilled water and saline water have been systematically studied. Besides, polymerization parameters such as the amount and kinds of initiators, crosslinking agents, neutralization concentration and monomers ratios have been investigated.

\section{Experimental}

\section{Materials}

Acrylic acid (Beijing Dongfang Chemical Plant) was purified by vacuum distillation. Acryl amide(AM), sodium hydroxide, potassium persulfate and $N, N^{\prime}$-methylene- bisacrylamide (NMBA) obtained from Tianjin Bodi Co were of reagent analytical grade. 2-acrylmido-2-methylpropanesulfonic acid (AMPS) supplied by Shandong Shouguang Chemical Plant was used as received. Triethylene glycol diacrylate (TEGDA) as crosslinking agent was also purchase from Beijing Dongfang Chemical Plant.

\section{Synthesis of AA-AM-AMPS copolymers}

AA and AMPS were neutralized by sodium hydroxide $(2.5 \mathrm{~mol} / \mathrm{L})$ to a proper neutralization degree at room temperature in a $100-\mathrm{mL}$ beaker. Then AM and crosslinking agent were added to the above solution. After that, the mixed solution was added to a $250-\mathrm{mL}$ threenecks flask equipped with a mechanical stirrer and a 
Fig. 3 Effect of crosslinking agent (TEGDA) concentration on the water absorbency of SAPs

Fig. 4 Effect of neutralization degree on the water absorbency of SAPs
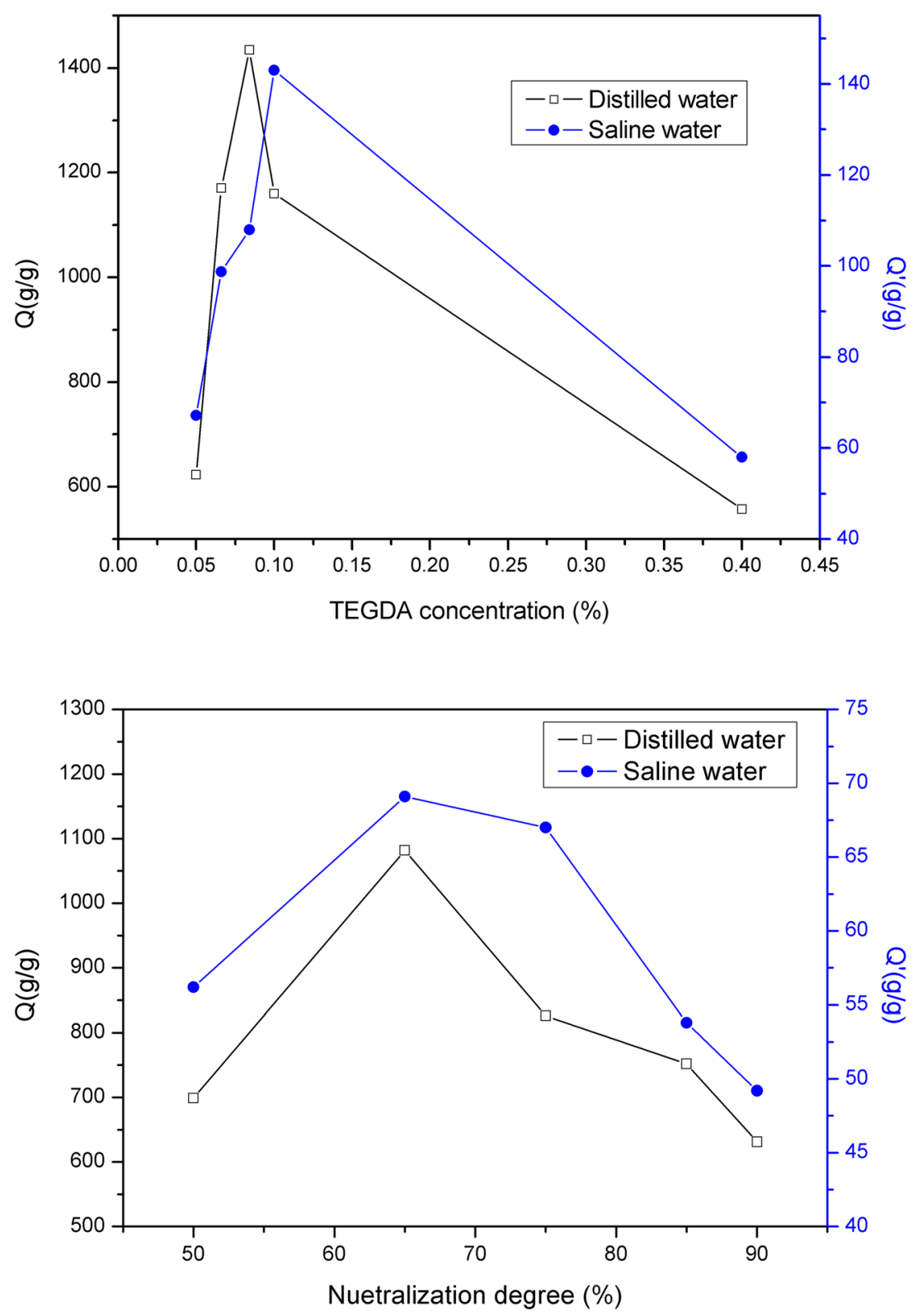

nitrogen line. The flask was put into a water-bath at $60{ }^{\circ} \mathrm{C}$ and the solution was flushed with nitrogen gas for $30 \mathrm{~min}$, and then the initiator solutions were added into the flask. The reactor was kept at $60{ }^{\circ} \mathrm{C}$ for $2 \mathrm{~h}$ and then heat to $70{ }^{\circ} \mathrm{C}$ for an additional $2 \mathrm{~h}$. The obtained product was cut into small pieces by hand.

\section{Measurement of properties}

All samples were dried in a vacuum oven at $100{ }^{\circ} \mathrm{C}$ until constant weight and they were smashed into particles with size between 100 and $200 \mu \mathrm{m}$. For the test of equilibrium water absorbency, nearly $0.1 \mathrm{~g}$ of dried SAP sample was put into a 300 mesh tea bag, then the bag was immersed in sufficient water for $24 \mathrm{~h}$ until the equilibrium water absorbency was reached. After that, the excessive water was filtrated by 200 mesh screen. The equilibrium water absorbency $(Q)$ was calculated by the following formula:

$Q=\left(m_{1}-m_{2}\right) / m_{2}-1$

where $m_{1}, m_{2}$ represents the weight of dried sample and swollen sample, respectively.

\section{Results and discussion}

\section{Influence of monomer ratio on water absorbency}

The influence of different monomer molar ratio (AA: AM: AMPS) on water absorbency was shown in Fig. 1. It could 
Fig. 5 Effect of initiator concentration on the water absorbency of SAPs
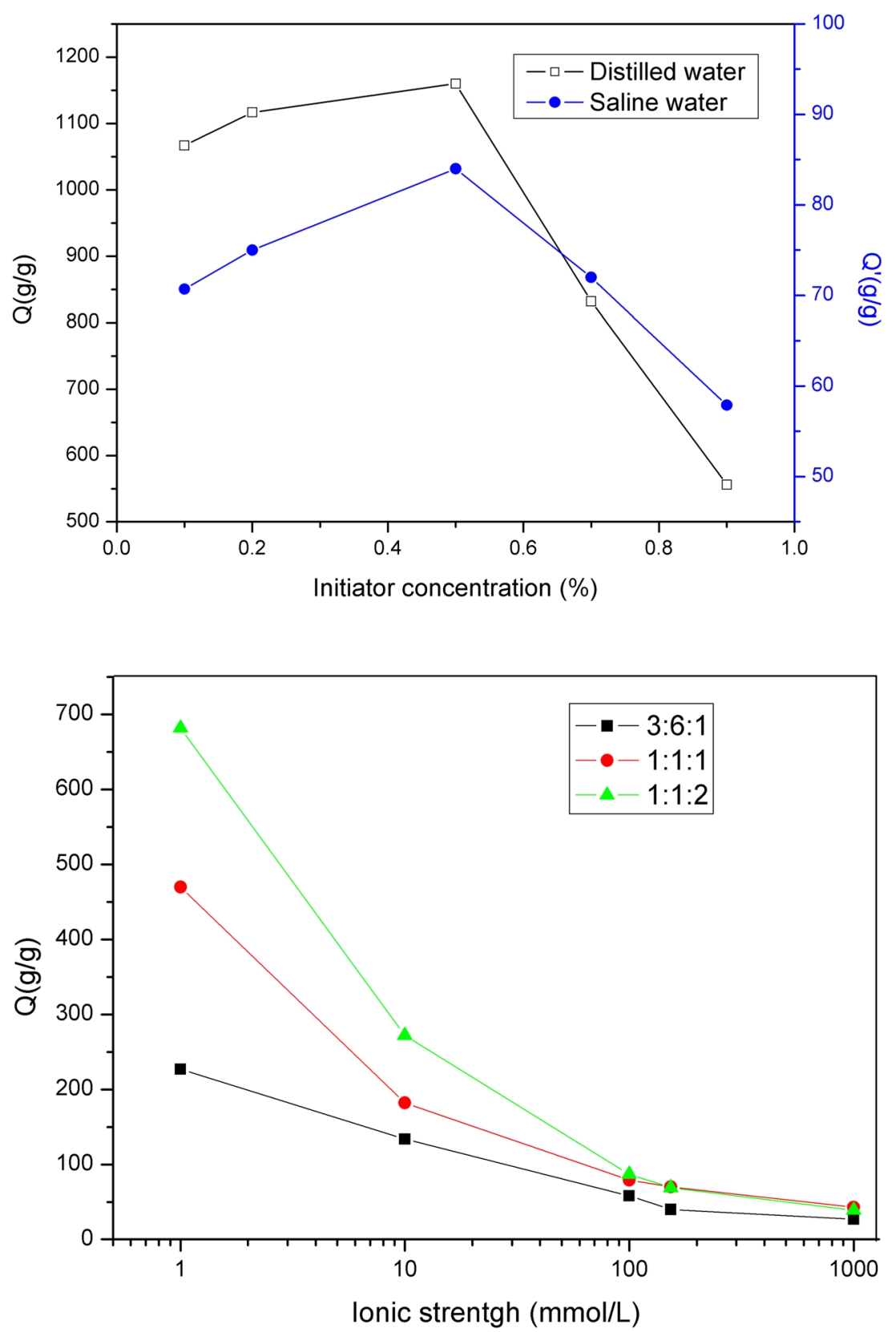

Fig. 6 Water absorbency of the poly(AA/AM/AMPS) SAPs in the various ionic strength of $\mathrm{NaCl}$ solution with different monomer ratio be seen that with the increase of amount of AMPS, the water absorbency of SAPs in saline water increased. This is because AMPS is an excellent salt-resistant monomer due to the high electric charge density of the functional group $\mathrm{SO}_{3} \mathrm{H}$. Then the $-\mathrm{NH}_{2}$ group is well protected by the large side group $-\mathrm{SO}_{3} \mathrm{H}$, so the salt ions have little influence on AMPS.

However, the trend of water absorbency in distilled water is a little different from that in saline water. As amount of AMPS was larger than 1/2 (molar ratio), the water absorbency decreased with the increase of AMPS. This is probably due to two reasons. In one hand, the hydrophilicity of $-\mathrm{SO}_{3} \mathrm{H}$ is worse than $-\mathrm{COOH}$ in distilled water, so the SAP with large amount of AMPS has worse water absorbency in distilled water; in another hand, SAPs with large amount of AMPS and small amount of AM are easier to shrink than the ones with small amount of AMPS and large amount of AM, leading to the decrease of water absorbency.

\section{Influence of crosslinking agent on water absorbency}

Figures 2 and 3 showed the water absorbency of SAPs with different crosslinking agent (NMBA and TEGDA) concentration. The swelling behavior of SAPs crosslinked by TEGDA is similar to those crosslinked by NMBA. The 
Fig. 7 Water absorbency of the poly(AA-AM-AMPS) SAPs in the various ionic strength of $\mathrm{NaCl}$ solution with different concentration of crosslinking agent

Fig. 8 Water absorbency of the poly(AA-AM-AMPS) SAPs in the various ionic strength of $\mathrm{NaCl}$ solution with different neutralization degree
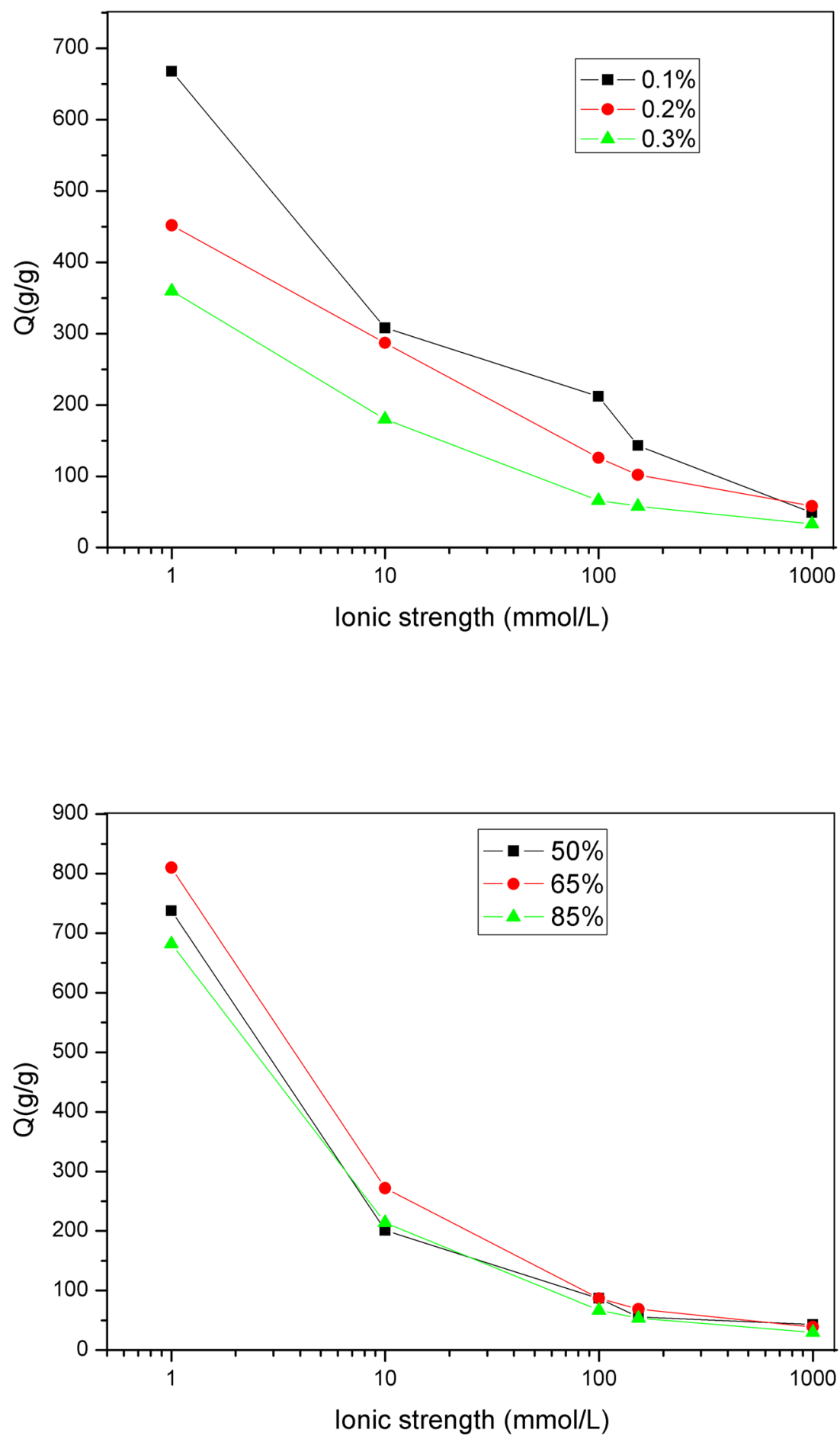

water absorbency increased as the NMBA concentration increased from 0 to $0.05 \%$ and then decreased as the concentration larger than $0.05 \%$. As is well known, the network size is determined by two factors: the crosslinking density and molecular weight between two crosslinking points (Mc), and the crosslinking density mainly depends on the concentration of the crosslinking agent. With the same kind of crosslinking agent, the network size is determined by the crosslinking density. At low concentration of the crosslinking agent, the crosslinking network of the SAP was not complete, and at higher concentration, the network size of SAP is too small, so there is an optimum amount of crosslinking agent for SAPs. 
It also can be seen that, when the molar concentration of crosslinking agent is $0.1 \%$, the water absorbency of SAP crosslinked by TEGDA was much larger than that of SAP crosslinked by NMBA. This is due to the different Mc of SAPs crosslinked by different crosslinking agents. Because the molecular chains length of TEGDA $\left(\mathrm{CH}_{2}=\mathrm{CHCOOCH}_{2}\right.$ $\mathrm{CHOCH}_{2} \mathrm{CHOCH}_{2} \mathrm{CHOOCCH}=\mathrm{CH}_{2}$ ) is obviously longer than that of NMBA $\left(\mathrm{H}_{2} \mathrm{C}=\mathrm{CHCONHCH}{ }_{2} \mathrm{NHCOCH}=\mathrm{CH}_{2}\right)$, at the same molar concentration of crosslinking agent, the network size of SAP is determined by the chain length of crosslinking agent and SAPs with larger network size have better water absorbency abilities.

\section{Influence of neutralization degree on water absorbency}

Figure 4 showed the influence of neutralization degree on the water absorbency of SAPs. It was obvious that when the neutralization degree increased from 50 to $65 \%$, the water absorbency increase; and the water absorbency decreased with neutralization degree increased continuously. In one hand, with the increase of neutralization degree, much $-\mathrm{SO}_{3} \mathrm{H}$ and $-\mathrm{COOH}$ were neutralized as $\mathrm{SO}_{3}{ }^{-}$and $-\mathrm{COO}^{-}$, leading to a strong electrostatic repulsion and a larger crosslinked network size, so the water absorbency increased; In another hand, with the neutralization degree increased continuously, many $-\mathrm{SO}_{3} \mathrm{H}$ and $\mathrm{COOH}$ groups were neutralized and the reactivity of these neutralized groups decreased obviously, so the reaction could not proceed completely, leading to a reduction in water absorbency.

\section{Influence of initiator concentration on water absorbency}

The influence of initiator concentration on the water absorbency was shown in Fig. 5. It was obvious that with the increase of initiator concentration, the water absorbency increased firstly and then decreased, i.e., there was an optimum value of the initiator concentration. This can be easily interpreted by basic theory of polymer chemistry. At low initiator concentration, the reaction could not proceed completely because of lack of free radicals supplied by the initiators, leading to a reduction in water absorbency. And at higher initiator concentration, the chain length would be shorter and the crosslinked network size would be small, leading to the decrease of water absorbency.

\section{Influence of ionic strength on water absorbency}

Figures 6, 7 and 8 showed the influence of ionic strength on the water absorbency of SAPs. The three figures all showed that with the increase of ionic strength in $\mathrm{NaCl}$ solutions, the water absorbencies of SAPs decreased. This is due to the cations $\left(\mathrm{Na}^{+}\right)$concentration in salt solution, as the $\mathrm{Na}^{+}$in the salt solution will neutralize the carboxylate and sulfonic group. With the increase of ionic strength in salt solution, the ionic osmotic pressure between the external solution and hydrogel decreases, so the water absorbency decreases. It also could be found that the water absorbency of the SAPs reach $58 \mathrm{~g} / \mathrm{g}$ at the ionic strength of $1000 \mathrm{mmol} / \mathrm{L}$, which proved that the SAPs had excellent salt-resistance property.

\section{Conclusion}

A new series of superabsorbent polymer, Poly(AA-AMAMPS) has been prepared in an aqueous solution using AA, AM and AMPS as monomers, potassium persulfate as initiator, $N, N^{\prime}$-methylenebiacrylamide (NMBA) or triethylene glycol diacrylate (TEGDA) as crosslinking agent.

There are optimum values for the polymerization parameters such as the concentration of the initiator, crosslinking agent, neutralization degree and monomer ratios.

The water absorbency of these SAPs decreased with the increase of the ionic strength in salt solution and the water absorbency of the SAPs can reach $58 \mathrm{~g} / \mathrm{g}$ at the ionic strength of $1000 \mathrm{mmol} / \mathrm{L}$, which proved that the synthesized SAPs had excellent salt-resistance property.

Acknowledgments This research is supported by National Science \& Technology Major Projects of China (2011ZX05011-004) and China Postdoctoral Science Foundation (2014M560573).

Open Access This article is distributed under the terms of the Creative Commons Attribution 4.0 International License (http:// creativecommons.org/licenses/by/4.0/), which permits unrestricted use, distribution, and reproduction in any medium, provided you give appropriate credit to the original author(s) and the source, provide a link to the Creative Commons license, and indicate if changes were made.

\section{References}

Chen Z, Liu M, Ma S (2005) Synthesis and modification of saltresistant superabsorbent polymers. React Funct Polym 62:85-92

Flory P (1953) Principles of Polymer Chemistry. 1st ed [M]. Cornell University Press, New York

Gosavi U, Deopurkar R, Ghole V (1999) Microbial degradation of superabsorbent HSPAN Gel by an indigenously isolated bacterial culture. Macromolecules 32:4264-4271

Guo M, Liu M (2005) Preparation and properties of a slow-release membrane-encapsulated urea fertilizer with superabsorbent and moisture preservation. Ind Eng Chem Res 44:4206-4211

Karadağ E, Saraydin D (2002) Swelling equilibria and dye adsorption studies of chemically crosslinked superabsorbent acrylamide/maleic acid hydrogels. Eur Polym J 38:2133-2141 
Lee W, Huang Y (2007) Swelling and antibacterial properties for the superabsorbent hydrogels containing silver nanoparticles. J Appl Polym Sci 106:1992-1995

Li A, Zhao Y, Wang A (2007) Study on superabsorbent composite. XII. Effect of ion-exchanged attapulgite on water absorbency of poly(acrylic acid)/attapulgite superabsorbent composites. J Appl Polym Sci 105:3476-3482

Mohan Y, Murthy P, Raju K (2005) Synthesis, characterization and effect of reaction parameters on swelling properties of acrylamide-sodium methacrylate superabsorbent copolymers. React Funct Polym 63:11-26

Murthy P, Mohan Y, Sreeramulu J, Raju K (2006) Semi-IPNs of starch and poly(acrylamide-co-sodium methacrylate): preparation, swelling and diffusion characteristics evaluation. React Funct Polym 66:1482-1493

Pourjavadi A, Ghasemzadeh H, Soleyman R (2007) Synthesis, characterization, and swelling behavior of alginate-g-poly(sodium acrylate)/kaolin superabsorbent hydrogel composites. J Appl Polym Sci 105:2631-2639
Vandamme TH, Lenourry A (2002) The use of polysaccharides to target drugs to the colon. Carbohyd Polym 48:219-231

Wu J, Lin J (2000) Synthesis and properties of starch-graftpolyacrylamide/clay superabsorbent composite. Macromol Rapid Comm 21:1032-1034

Xu K, Wang J, Xiang S (2007) Polyampholytes superabsorbent nanocomposites with excellent gel strength. Compos Sci Technol 67:3480-3486

Yilmaz S, Kul D (2007) Synthesis of a novel crosslinked superabsorbent copolymer with diazacyclooctadecane crown ether and its sorption capability. Eur Polym J 43:1923-1932

Zhang J, Liu R, Li A (2006) Preparation, swelling behaviors, and slow-release properties of a poly(acrylic acid-co-acrylamide)/sodium humate superabsorbent composite. Ind Eng Chem Res 45:48-53

Zou X (2002) Super Absorbents. 1st ed [M]. Chemical Industry Press, Beijing 